\title{
High-Q resonances with low azimuthal indices in all-dielectric high-index nanoparticles
}

Kirill L. Koshelev, Andrey A. Bogdanov, Zarina F. Sadrieva, Kirill B. Samusev, Mikhail V. Rybin, et al.

Kirill L. Koshelev, Andrey A. Bogdanov, Zarina F. Sadrieva, Kirill B. Samusev, Mikhail V. Rybin, Mikhail F. Limonov, Yuri S. Kivshar, "High-Q resonances with low azimuthal indices in all-dielectric high-index nanoparticles," Proc. SPIE 10343, Metamaterials, Metadevices, and Metasystems 2017, 103430F (24 August 2017); doi: 10.1117/12.2272375

Event: SPIE Nanoscience + Engineering, 2017, San Diego, California, United States 


\title{
High-Q resonances with low azimuthal indices in all-dielectric high-index nanoparticles
}

\author{
Kirill L. Koshelev ${ }^{\mathrm{a}, \mathrm{d}}$, Andrey A. Bogdanov ${ }^{\mathrm{a}, \mathrm{b}}$, Zarina F. Sadrieva ${ }^{\mathrm{a}}$, Kirill B. Samuseva, \\ Mikhail V. Rybin ${ }^{\mathrm{a}, \mathrm{b}}$, Mikhail F. Limonov ${ }^{\mathrm{a}, \mathrm{b}}$, and Yuri S. Kivshar ${ }^{\mathrm{a}, \mathrm{c}}$

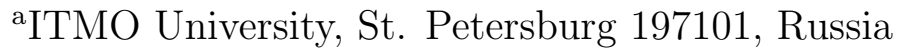 \\ ${ }^{\mathrm{b}}$ Ioffe Institute, St. Petersburg 194021, Russia \\ 'Nonlinear Physics Centre, Australian National University, Canberra ACT 2601, Australia \\ ${ }^{\mathrm{d}}$ The Academic University, St. Petersburg 194021, Russia
}

\begin{abstract}
Recently, a novel class of high-Q optical resonators based on all-dielectric subwavelength nanoparticles with high refractive index has been proposed [M. V. Rybin, et al, arXiv:1706.02099, 2017]. Here we study a complex spectrum of such nanoscale resonators by means of the resonant-state expansion, treating the problem as a nonHermitian eigenproblem. We show that the high-Q features can be described within the mechanism of external coupling of open channels via the continuum. For ceramic resonators with permittivity $\varepsilon=40$, we demonstrate that the quality factor of a trapped mode with a low azimuthal index could reach the value $Q=10^{4}$.
\end{abstract}

Keywords: all-dielectric, high-Q resonator, subwavelength, Fano resonance, non-Hermitian.

\section{INTRODUCTION}

Resent progress in all-dielectric nanophotonics opens many opportunities for the enhanced resonant phenomena in nanoscale meta-optics. ${ }^{1}$ However, usually dielectric resonators demonstrate low values of the quality factor (Q-factor) and low efficiencies of the Purcell effect for Mie-type resonant modes. ${ }^{2}$

Recently, a novel approach to achieve high-Q optical resonators based on all-dielectric high-index nanoparticles has been proposed. ${ }^{3}$ It was shown that high-quality modes of a finite-size nanorod can be excited due to a hybridization of resonant Mie and Farby-Perot modes with low azimuthal indices which leads to substantial suppression of far-field radiation. Regime of giant Q-factors can be directly observed in terms of parameters of the scattered field which is described by a Fano profile.

In this paper, we investigate the spectrum of a nanoscale dielectric nanorod in terms of its complex eigenfrequencies. We apply the resonant-state expansion ${ }^{4}$ (RSE) to study a non-Hermitian eigenproblem with the boundary conditions of outgoing waves. The rigorous non-Hermitian approach allows to explain the high-Q features as a result of external coupling of resonator's open channels via the continuum. We focus on the case of ceramic resonators with permittivity $\varepsilon=40$ and demonstrate that the quality factor of a mode with a low azimuthal index could reach $Q=10^{4}$.

\section{RESONANT STATE EXPANSION}

Maxwell's equations for a finite-size dielectric nanorod are not exactly solvable. ${ }^{5}$ Precise calculation of spectrum of a resonant nanoscale nanorod by means of standard numerical techniques (e.g., FEM, FDTD) requires large computational resources, and it can suffer from significant numerical errors. ${ }^{6,7}$

In order to avoid these problems, a rigorous perturbative method in electrodynamics, (RSE), was recently developed for three-dimensional open optical systems. ${ }^{4}$ For the first time to our knowledge, we apply this approach to study a spectrum $\Omega_{j}$ of a dielectric nanorod which is found by expanding the cylinder fields $\mathbf{E}_{j}, \mathbf{H}_{j}$

Further author information: (Send correspondence to K.K.L.)

K.K.L.: E-mail: ki.koshelev@gmail.com, Telephone: 79215588641

Metamaterials, Metadevices, and Metasystems 2017, edited by Nader Engheta, Mikhail A. Noginov, Nikolay I. Zheludev, Proc. of SPIE Vol. 10343, 103430F

(c) 2017 SPIE · CCC code: 0277-786X/17/\$18 - doi: 10.1117/12.2272375

Proc. of SPIE Vol. 10343 103430F-1 
(a)

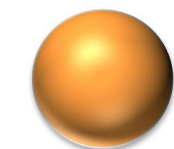

$\mathbf{E}_{n}^{(0)}, \mathbf{H}_{n}^{(0)}, \Omega_{n}^{(0)}$
Resonant State Expansion
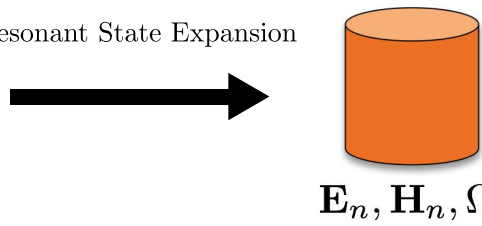

$\mathbf{E}_{n}, \mathbf{H}_{n}, \Omega_{n}$ (b)

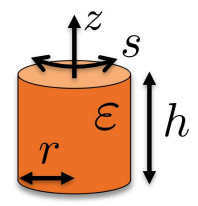

Figure 1. (a) Schematic illustration of the resonant-state expansion for a finite dielectric nanorod. (b) Model of a dielectric nanorod with the height $h$ and the radius $r$ which is made of material with a dielectric function $\varepsilon . s=0, \pm 1, .$. is the azimuthal index enumerating eigenmodes of the resonator.

over the basis of an analytically solvable homogeneous dielectric sphere $\mathbf{E}_{n}^{(0)}, \mathbf{H}_{n}^{(0)}$, as shown schematically in Fig. 1(a).

Resonant states of an homogeneous dielectric sphere with a scalar time-independent tensor of dielectric permittivity $\varepsilon(\mathbf{r})$ and permeability $\mu=1$ are defined as the eigenvectors of the Maxwell's wave equation with the boundary conditions of outgoing waves

$$
\nabla \times \nabla \times \mathbf{E}_{n}^{(0)}=\varepsilon(\mathbf{r}) \frac{\left(\Omega_{n}^{(0)}\right)^{2}}{c^{2}} \mathbf{E}_{n}^{(0)} .
$$

Resonants states of a dielectric nanorod satisfy the perturbed equation

$$
\nabla \times \nabla \times \mathbf{E}_{j}=[\varepsilon(\mathbf{r})+\Delta \varepsilon(\mathbf{r})] \frac{\Omega_{j}^{2}}{c^{2}} \mathbf{E}_{j},
$$

where $\Delta \varepsilon(\mathbf{r})$ is a perturbation that transforms the sphere into an inscribed cylinder.

The outgoing boundary conditions make the eigenvalue problem inherently non-Hermitian. Eigenvectors $\mathbf{E}_{n}^{(0)}$ grow exponentially at large distances, and they cannot be normalized by the standard approach for Hermitian systems. However, $\mathbf{E}_{n}^{(0)}$ form a complete set inside the region of a dielectric sphere which can be used as a basis for our problem. The proper normalization can be derived from general formalism ${ }^{4}$

$$
\delta_{n m}=\int \varepsilon(\mathbf{r}) \mathbf{E}_{n}^{(0)} \mathbf{E}_{m}^{(0)} d V+\mathbb{S}_{n m}\left[\Omega_{n}^{(0)}, \Omega_{m}^{(0)}\right],
$$

where $\mathbb{S}_{n m}$ is a surface term which compensates the diverging contribution coming from the volume integal.

Expanding the perturbed field

$$
\mathbf{E}_{j}=\sqrt{\Omega_{j}} \sum_{n} \frac{\alpha_{n}^{j}}{\sqrt{\Omega_{n}^{(0)}}} \mathbf{E}_{n}^{(0)},
$$

one can arrive at the matrix equation ${ }^{4}$

$$
\hat{\Theta}\left|\alpha^{j}\right\rangle=\frac{1}{\Omega_{j}}\left|\alpha^{j}\right\rangle .
$$

Here $\hat{\Theta}$ is the master operator

$$
\hat{\Theta}=\left[\left(\hat{\Omega}^{(0)}\right)^{-1}+\hat{V}\right],
$$

an the matrix elements of the perturbation $V_{n m}$ are defined as

$$
V_{n m}=\frac{1}{2} \frac{\int d \mathbf{r} \Delta \varepsilon(\mathbf{r}) \mathbf{E}_{n}(\mathbf{r}) \cdot \mathbf{E}_{m}(\mathbf{r})}{\sqrt{\Omega_{n}^{(0)}} \sqrt{\Omega_{m}^{(0)}}} .
$$

We consider a model of a finite-size all-dielectric nanorod made of material with isotropic permittivity $\varepsilon$. The parameters of the cylinder are shown in Fig. 1(b). Since the RSE is the Brillouin-Wigner perturbation theory, the numerical accuracy is defined only by the size of the basis set $N$. 


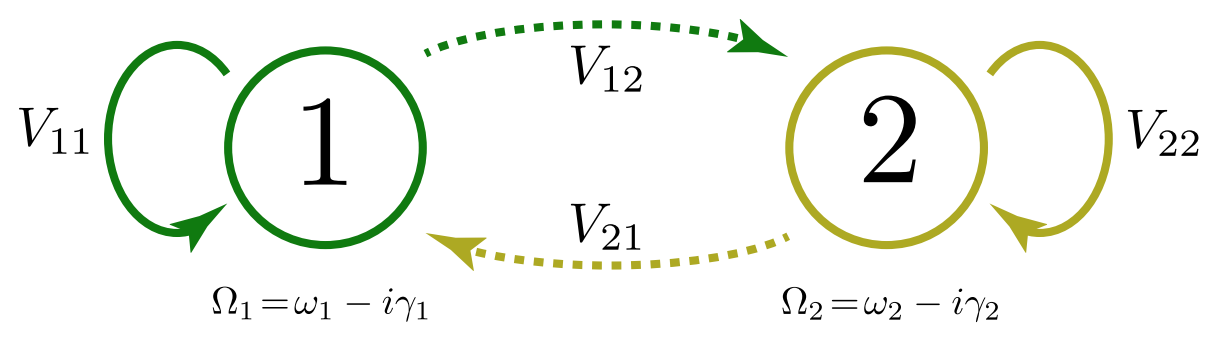

Figure 2. Schematic of interaction between two channels of an open electromagnetic system. Individual couplings of channels to the continuum $V_{11}, V_{22}$ are shown by solid lines. External couplings between channels via the continuum $V_{12}, V_{21}$ are depicted by dashed lines.

\section{AVOIDED RESONANCE CROSSINGS: EXTERNAL COUPLING OF RESONANT MODES VIA THE CONTINUUM}

Coupling between the modes in open electromagnetic cavities or resonators is determined by the structure parameters such as geometrical size, shape or material. Variation of one of the parameters can entail avoided resonance crossings (ARC) behaviour of spectrum. For the case of closed or conservative systems an anticrossing regime is well-studied, ${ }^{8}$ resonance coupling is described by a strong hybridization of modes which causes a repulsion of dispersion curves from each other.

In open systems the physics lying behind the ARC regime is richer because both weak and strong coupling is possible. The qualitative behaviour can be understood in terms of a toy model, a two level system, which is described by the simplified master operator $\hat{\Theta}$ from Eq. (6)

$$
\hat{\Theta}(\xi)=\left[\begin{array}{cc}
1 / \Omega_{1}+V_{11}(\xi) & V_{12}(\xi) \\
V_{21}(\xi) & 1 / \Omega_{2}+V_{22}(\xi)
\end{array}\right]
$$

where $\xi$ is a parameter of resonator which continuous variation entails the ARC behaviour of the spectrum.

Resonator eigenmodes represent open channels which can be coupled to the continuum individually by $V_{11}, V_{22}$ as well as can couple each other externally via continuum by $V_{12}, V_{21}$. The scheme of interaction is shown in Fig. 2. Eigenvalues of diagonalized matrix $1 / \Omega_{ \pm}$are readily given as

$$
\frac{1}{\Omega_{ \pm}}=\frac{\left(1 / \Omega_{1}+1 / \Omega_{2}+V_{11}+V_{22}\right)}{2} \pm\left[\frac{\left(1 / \Omega_{1}-1 / \Omega_{2}+V_{11}-V_{22}\right)^{2}}{4}+V_{12} V_{21}\right]^{1 / 2} .
$$

In a general case $V_{12} \neq V_{21}^{*}$ one of states $( \pm)$ has an increased lifetime at the center of the ARC and the another one has a decreased decay rate accordingly. This phenomenon occurs due to the destructive interference of channels and can lead to a dramatic increase of Q-factor. ${ }^{9-13}$ It should be noticed that the referred papers report about the enlargement of Q-factor in systems of coupled resonators and cavities, and the first to our knowledge manifestation of such effect for a finite-size single subwavelength resonator was studied in [3].

\section{RESULTS OF THE RSE CALCULATIONS}

The model described above is applicable for dielectric cylinders of various compounds and proportions. As an example, let us consider a high-index ceramic nanorod with permittivity $\varepsilon=40$. We calculate the spectrum of such nanocylinder by using the RSE method. We investigate how real and imaginary part of modes depend on the cylinder aspect ratio $\xi=r / h$.

The rotational and mirror $(z \rightarrow-z)$ symmetries of system allow us to classify the modes with respect to their azimuthal index $s=0, \pm 1, \pm 2, .$. and to their parity $( \pm)$. The modes of different symmetry are not mixed when $\xi$ changes because the transition $\xi \rightarrow \xi+\Delta \xi$ does not break system symmetries. For the sake of simplicity, we focus only on the even modes with $s=0$ and $s= \pm 1$. 


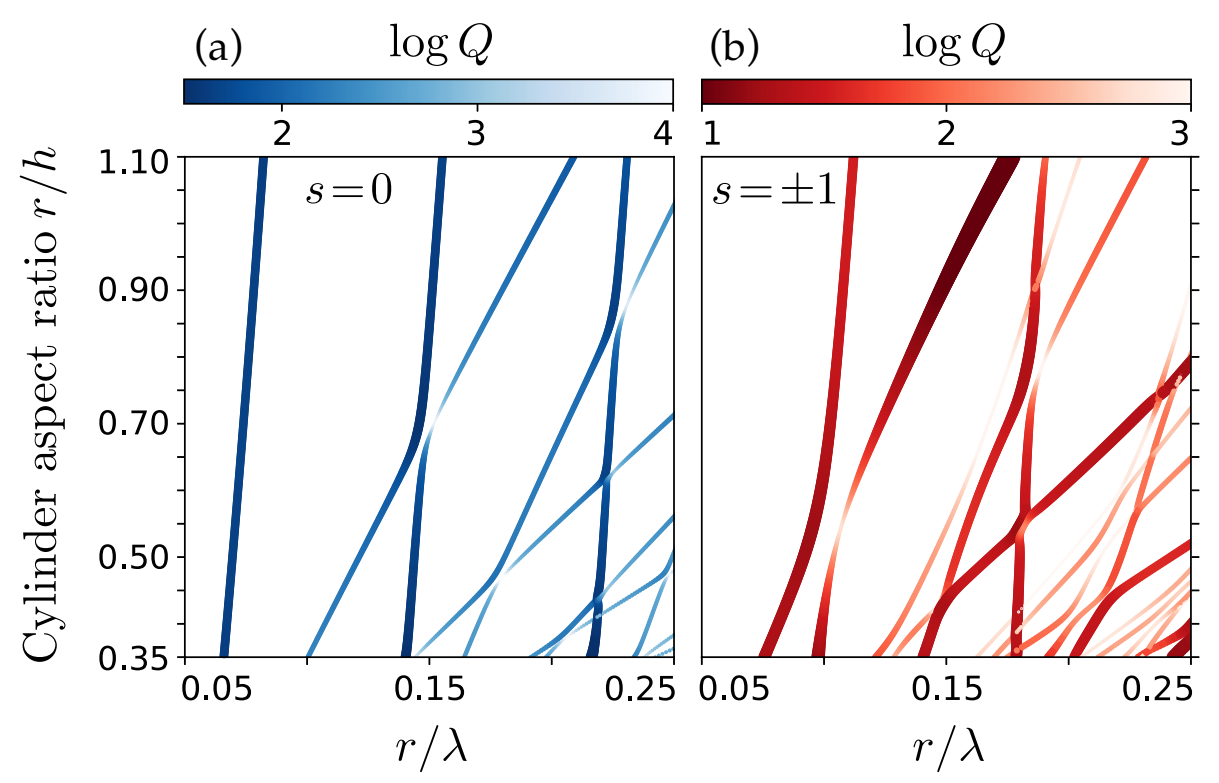

Figure 3. Dependence of the complex spectrum of eigenmodes with (a) $s=0$ and (b) $s= \pm 1$, which are even with respect to $(z \rightarrow-z)$ symmetry, vs. the cylinder aspect ratio $r / h$. Here $r / \lambda$ is a dimensionless parameter proportional to the real part of mode eigenfrequency. The logarithm of the mode quality factor $Q$ is shown in color. The linewidth is proportional to $Q^{-1}$. The calculations are performed by using the RSE method.
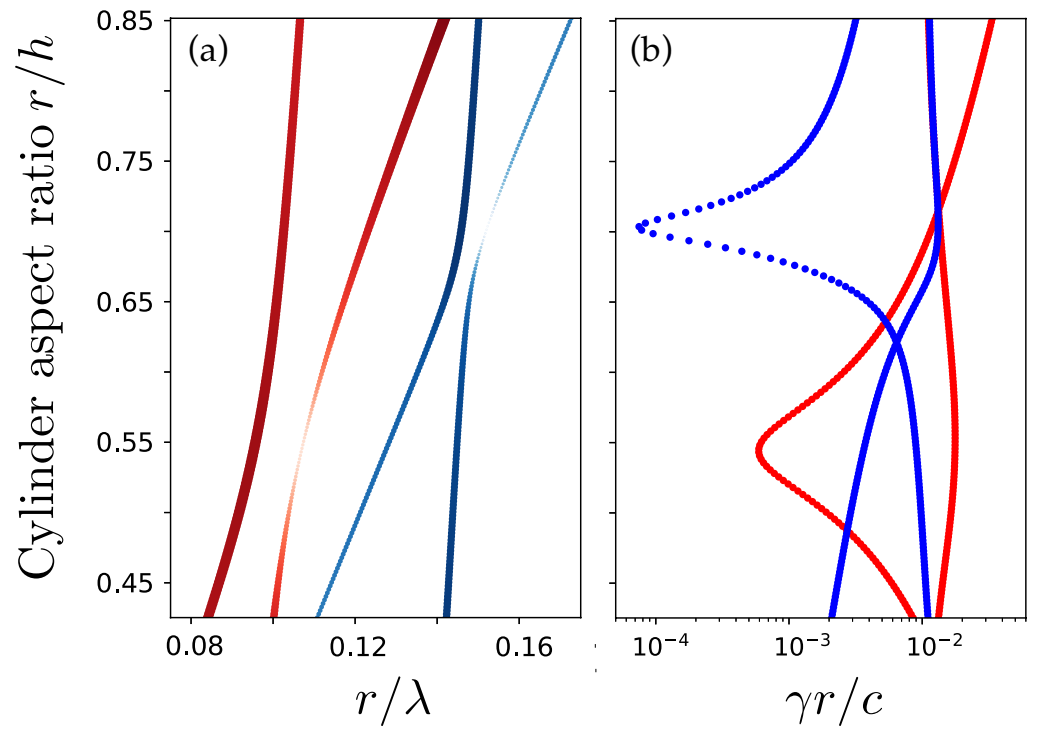

Figure 4. Dependence of (a) real and (b) imaginary part of spectrum of two first ARC. Colorbar is the same as in Fig. 3. Modes with $s=0$ and $s= \pm 1$ are shown by blue and red lines, respectively. The calculations are performed by using the RSE method.

The spectrum $\Omega=\omega-i \gamma$ of even eigenmodes with azimuthal indices 0 and \pm 1 is shown in Fig. 3(a) and Fig. 3(b), respectively. In our calculations we truncate the size of the basis set to $N=2000$ which is sufficient for convergence. Here $\lambda=2 \pi / \omega$ is the wavelength and $Q=\omega / 2 \gamma$ is mode quality factor. Color depicts the magnitude of $Q$ and linewidth is plotted to be proportional to $Q^{-1}$. One can clearly observe multiple avoided resonance crossings which lead to a dramatical enlargement of Q-factor of modes. Therefore, the predictions of the simplified two-level model Eq. (8) are proved by the rigorous calculations. One can see that the maximal Q-factor is of order of $10^{4}$, which is two orders of magnitude higher than the Q-factor of the low azimuthal index modes of a spherical resonator with $\varepsilon=40$. 
Next we investigate the behaviour of $\gamma$ of two first ARC from Fig. 3 in detail. The real part of the eigenfrequencies is shown in Fig. 4(a). The dependence of the imaginary part of the eigenfrequency is plotted in Fig. 4(b). The lines $\gamma(r / h)$ cross, whereas the lines $\omega(r / h)$ exhibit an anticrossing. Such behaviour is the peculiarity of strong coupling regime that can occur for spectrum of open resonators. One can observe that the damping rate $\gamma$ of modes with $s=0$ is at least an order of magnitude smaller than for modes with other $s$. This can be explained by the fact that the modes with $s=0$ are symmetric with respect to any rotation around cylinder axis. Hence their individual coupling with the free-space continuum is highly suppressed which explains the small values of $\gamma$.

\section{CONCLUSION}

In summary, we have studied the spectrum of a finite-size dielectric nanorod by means of the resonant-state expansion. We have explained the high- $\mathrm{Q}$ features as a result of external coupling of resonator's open channels via the continuum within a simple model of two level non-Hermitian system. We have calculated the dependence of the real and imaginary parts of frequencies of ceramic nanoscale cylinder with permittivity $\varepsilon=40$ on cylinder aspect ratio $r / h$. Particularly, we have demonstrated that the quality factor of a mode with the azimuthal index $s=0$ could reach the value $Q=10^{4}$. The modes with $s=0$ have the lowest damping rate because of their symmetric angular distribution which implies that the coupling with the free-space continuum is highly suppressed.

\section{ACKNOWLEDGMENTS}

This work has been supported by the Ministry of Education and Science of the Russian Federation (3.1668.2017/ 4.6) and the Russian Foundation for Basic Research (16-02-00461, 16-37-60064, 17-02-01234).

\section{REFERENCES}

[1] Kuznetsov, A. I., Miroshnichenko, A. E., Brongersma, M. L., Kivshar, Y. S., and Luk'yanchuk, B., "Optically resonant dielectric nanostructures," Science 354(6314), aag2472 (2016).

[2] Krasnok, A., Glybovski, S., Petrov, M., Makarov, S., Savelev, R., Belov, P., Simovski, C., and Kivshar, Y., "Demonstration of the enhanced purcell factor in all-dielectric structures," Applied Physics Letters 108(21), 211105 (2016).

[3] Rybin, M. V., Koshelev, K. L., Sadrieva, Z. F., Samusev, K. B., Bogdanov, A. A., Limonov, M. F., and Kivshar, Y. S., "High-q supercavity modes in subwavelength dielectric resonators," arXiv preprint arXiv:1706.02099 (2017).

[4] Doost, M., Langbein, W., and Muljarov, E. A., "Resonant-state expansion applied to three-dimensional open optical systems," Physical Review A 90(1), 013834 (2014).

[5] Stratton, J. A., [Electromagnetic theory], John Wiley \& Sons (2007).

[6] Bayliss, A., Goldstein, C. I., and Turkel, E., "On accuracy conditions for the numerical computation of waves," Journal of Computational Physics 59(3), 396-404 (1985).

[7] Ihlenburg, F., Babuska, I., and Sauter, S., "Reliability of finite element methods for the numerical computation of waves," Advances in Engineering Software 28(7), 417-424 (1997).

[8] von Neumann, J. and Wigner, E., "Über merkwürdige diskrete eigenwerte," Phys. Z. 30 (1929).

[9] Boriskina, S. V., "Theoretical prediction of a dramatic q-factor enhancement and degeneracy removal of whispering gallery modes in symmetrical photonic molecules," Optics letters 31(3), 338-340 (2006).

[10] Boriskina, S. V., "Coupling of whispering-gallery modes in size-mismatched microdisk photonic molecules," Optics letters 32(11), 1557-1559 (2007).

[11] Yang, Y.-D. and Huang, Y.-Z., "Mode analysis and $q$-factor enhancement due to mode coupling in rectangular resonators," IEEE journal of quantum electronics 43(6), 497-502 (2007).

[12] Song, Q. and Cao, H., "Improving optical confinement in nanostructures via external mode coupling," Physical review letters 105(5), 053902 (2010).

[13] Carmon, T., Schwefel, H. G., Yang, L., Oxborrow, M., Stone, A. D., and Vahala, K. J., "Static envelope patterns in composite resonances generated by level crossing in optical toroidal microcavities," Physical Review Letters 100(10), 103905 (2008). 